\title{
SIMPLE GROUPS OF SMALL ENGEL DEPTH
}

\author{
Rolf Brandl and Daniela B. Ni kolova
}
It is proved that the simple group $P S L(2, q)$ satisfies a law $\left[x, 3^{y}\right]=\left[x, s^{y}\right], s>3$, if and only if $q=4,5,8$.

\section{Introduction.}

Every finite group $G$ satisfies a law

$$
[x, y, y]=[x, y] \text {, for some } s>r \text {, }
$$

where

$$
\begin{aligned}
& {[x, y]=x,\left[x, n^{y}\right]=\left[\left[x, n-1^{y}\right], y\right] \text {, for } n \geq 1 .} \\
& \text { If } r \text { is chosen minimal with respect to this property, then } r \text { is }
\end{aligned}
$$
called the Engel depth of $G$ ([1]). It was proved in [2], [1] and [5] that finite groups of Engel depth $r \leq 2$ are soluble. However, there are nonabelian simple groups of depth three. For example, the groups $P S L(2,4)$ and $\operatorname{PSL}(2,8)$ have this property, as can be seen from the following table exhibiting the minimal parameters $r, s$ for some groups

Received 19 June 1985. The second author wishes to express her gratitude to Professor $\mathrm{H}$. Heineken for the excellent atmosphere offered to her in the University of wïrzburg and to the DAAD-foundation for making this collaboration possible.

Copyright clearance Centre, Inc. Serial-fee code: 0004-9727/86 $\$ A 2.00+0.00$. 
$G=\operatorname{PSL}(2, q):$

\begin{tabular}{l|c|c|c|c|c|c}
$q$ & 4 & 7 & 8 & 9 & 11 & 13 \\
\hline$\left.\right|_{G} \mid$ & 60 & 168 & 504 & 360 & 660 & 1092 \\
\hline$r$ & 3 & 4 & 3 & 4 & 6 & 7 \\
\hline$s$ & 63 & 172 & 129 & 124 & 1986 & 2191 \\
\hline
\end{tabular}

The computations have been performed on a $T R 440$ at the Rechenzentrum der Universitat wurzburg and on an EC 1040 at the Computing Centre of the Bulgarian Academy of Sciences, Sofia. Note that there seems to be a relationship between $s-r$ and the order of the group.

We are interested in finite simple groups contained in the class $V_{p}$ of all finite groups of Engel depth $\leq r$. In this context a theorem of H. Heineken and P. M. Neumann [3] deserves attention, stating that any nontrivial variety of groups contains only finitely many of the finite simple groups $P S L(2, q)$ or $S z(q)$. The classes $V_{p}$ are not varieties, but we feel that they should have some common properties with varieties. In particular, we conjecture that any $V_{r}$ contains only finitely many nonabelian simple groups.

Here we prove the following

THEOREM. Let $G=\operatorname{PSL}(2, q)$, for $q \geq 4$. Then $G \in V_{3}$ if and only if $q=4,5$, or 8 .

There is some evidence that the groups mentioned above are the only finite simple groups in $V_{3}$. For example, the smallest Suzuki group $S z(8)$ has Engel depth at least 11.

\section{Proof of the Theorem}

For the proof of the Theorem we need to construct elements $x, y \in G$, such that $\left[x, z^{y}\right] \neq[x, y]$, for all $s>3$. In computational experiments such elements abound, but for a general proof some care is needed. Our choice is motivated by the following 
Example. Let $G=\operatorname{PSL}(2,9)$. For all $x, y \in G$, such that $|y| \neq 4$, we have $\left[x, z^{y}\right]=\left[x, 63^{y}\right]$. Nevertheless, there exist $x \in G$

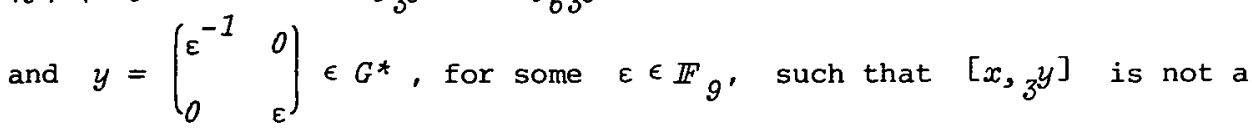
transvection, but $[x, y]$ is a transvection, for all $s>3$.

The following result exhibits elements of Engel depth three:

LEMMA 1. Let $q$ be a prime power and let $\lambda, s, u, \varepsilon \in \mathbb{F}_{q}$ be such that

$$
\lambda \operatorname{su}\left(1-\varepsilon^{2}\right)=1 \text { and } \varepsilon \neq 0 \text {. }
$$

Let $z_{1}=\left(\begin{array}{cc}\lambda \varepsilon & s \\ \varepsilon^{2} \lambda u & u\end{array}\right)$ and $y=\left(\begin{array}{cc}\varepsilon^{-1} & 0 \\ 0 & \varepsilon\end{array}\right)$.

Then $\left[z_{1}, 2^{y}\right]=\left(\begin{array}{cc}\varepsilon^{-2} & \lambda^{-1}\left(1-\varepsilon^{4}\right) \\ 0 & \varepsilon^{2}\end{array}\right)$.

If $\varepsilon^{2} \neq \pm 1$, then $\left[z_{1}, 2^{y}\right] \neq\left[z_{1}, s^{y}\right]$, for all $s \geq 3$

Proof. The statement follows from a straight forward calculation, as $\left[z_{1}, s\right]$ are all transvections, for $s \cdot \geq 3$.

The next result reduces the proof of the Theorem to solving a quadratic equation in the field of $q$ elements.

LEMMA 2. If the equation

$$
\left(1-\varepsilon^{2}\right) u^{2}+\left(\varepsilon^{4}-1\right) u+\varepsilon^{2}=0
$$

has a solution $u, \varepsilon \in \mathbb{F}_{q}$, where $\varepsilon^{2} \neq 0$, \pm 1 , then there exists $z_{0} \in \operatorname{PSL}(2, q)$, such that $\left[z_{0}, 3^{y}\right] \neq\left[z_{0}, s^{y}\right]$, for all $s \geq 4$.

Proof. Let $z_{1}$ and $y$ be as in Lemma 1 . According to it, it suffices to find $z_{0} \in \operatorname{PSL}(2, q)$, such that $\left[z_{0}, y\right]=z_{1}$. Let

* Henceforth, we shall identify $2 \times 2$-matrices in $S L(2, q)$ with their images in $\operatorname{PSL}(2, q)$. 
$z_{0}=\left(\begin{array}{ll}a & b \\ c & d\end{array}\right)$. Then $\left[z_{0}, y\right]=z_{1}$ is equivalent to $y^{-1} z_{0} y=z_{0} z_{1}$,

that is to

$$
\left(\begin{array}{cc}
a & \varepsilon^{2} b \\
\varepsilon^{-2} c & d
\end{array}\right)=\left(\begin{array}{cc}
\lambda s a+\varepsilon^{2} \lambda u b & s a+u b \\
\lambda s c+\varepsilon^{2} \lambda u d & s c+u d
\end{array}\right) .
$$

This in turn, is equivalent to the following system of linear equations for $a, b, c, d$ :

$$
\begin{aligned}
(\lambda s-1) a+\varepsilon^{2} \lambda u b & =0 \\
s a+\left(u-\varepsilon^{2}\right) b & =0 \\
\left(\lambda s-\varepsilon^{-2}\right) c+\varepsilon^{2} \lambda u d & =0 \\
s c+(u-1) d & =0 .
\end{aligned}
$$

There exists a solution $(a, b) \neq(0,0)$ if and only if

$$
D_{1}=\left|\begin{array}{cc}
\lambda s-1 & \varepsilon^{2} \lambda u \\
s & u-\varepsilon^{2}
\end{array}\right|=1-u+\varepsilon^{2}(1-\lambda s)=0 .
$$

Similarly, there exists a nontrivial solution $(c, d)$ if and only if

$$
D_{2}=\left|\begin{array}{cc}
\lambda s-\varepsilon^{-2} & \varepsilon^{2} \lambda u \\
s & u-1
\end{array}\right|=\varepsilon^{-2}\left(1-u+\varepsilon^{2}(1-\lambda s)\right)=0 .
$$

Both conditions are equivalent to

$$
1-u+\varepsilon^{2}(1-\lambda s)=0 \text {. }
$$

Using the condition (C) from Lemma 1, we get that (S) has a nontrivial solution if and only if

$$
\left(1-\varepsilon^{2}\right) u^{2}+\left(\varepsilon^{4}-1\right) u+\varepsilon^{2}=0
$$

has a solution $u, \varepsilon \in I_{q}$, where $\varepsilon^{2} \neq 0, \pm 1$. Then the parameters $\lambda$ and $s$ can be determined from (C).

Moreover, it follows from $(S)$ that the vectors $(a, b)$ and $\left(\lambda s-1, \varepsilon^{2} \lambda u\right)$ are perpendicular with respect to the usual scalar product, so are the vectors $(c, d)$ and $\left(\lambda s-\varepsilon^{-2}, \varepsilon^{2} \lambda u\right)$. Hence, if all nontrivial solutions $(a, b)$ and $(c, d)$ of $(S)$ were linearly dependent, 
then $\left(\lambda s-1, \varepsilon^{2} \lambda u\right)$ and $\left(\lambda s-\varepsilon^{-2}, \varepsilon^{2} \lambda u\right)$ would be linearly dependent. But (C) implies that $\varepsilon^{2} \lambda u \neq 0$ and so $\lambda s-1=\lambda s-\varepsilon^{-2}$, contradicting our assumption $\varepsilon^{2} \neq 1$. So, there exists $z_{0} \in G L(2, q)$ with $\operatorname{det}\left(z_{0}\right) \neq 0$ and $\left[z_{0}, y\right]=z_{1}$. Multiplying the first row of $z_{0}$ by the inverse of $\operatorname{det}\left(z_{0}\right)$, we get an element of $\operatorname{PSL}(2, q)$ with the required properties. We now solve $(E)$ in $\mathbb{F}_{q^{\cdot}}$ Let

$$
F(x, y)=\left(1-y^{2}\right) x^{2}+\left(y^{4}-1\right) x+y^{2}
$$

and let $N$ be the number of pairs $(u, \varepsilon) \in \mathbb{F} q \mathbb{F}_{q}$ such that $F(u, \varepsilon)=0$. A simple appeal to Eisenstein's Theorem shows that $F$ is absolutely irreducible. From a well-known Theorem of A. Weil in Algebraic Geometry (see [6;p. 449]) it follows that $|N-q| \leq 12 \sqrt{q}+5$. There are at most six "trivial" solutions $u, \varepsilon$ of $(E)$ where $\varepsilon^{2}=0, \pm 1$. Hence if $q \geq 169$, we get $N \geq 7$ and so (E) has at least one "nontrivial" solution.

We now deal with the remaining cases. First let $q$ be odd. Then (E) has a solution $u \in \mathbb{F} q$ if and only if its discriminant

$$
D(\varepsilon)=\left(\varepsilon^{4}-2 \varepsilon+1\right)\left(\varepsilon^{4}+2 \varepsilon+1\right)
$$

is a square in $\mathbb{F}_{q}$. Hence our problem is reduced in this case to proving that there exists $\varepsilon \in \mathbb{F}_{q}, \varepsilon^{2} \neq 0, \pm 1$ such that $D(\varepsilon)$ is a square. If $q=p^{f}$ with $7 \leq p \leq 168$, then a direct calculation shows that such $\varepsilon \in \mathbb{F}_{p}$ exists.

We consider the cases $p=2,3,5$ separately. Here the problem is more complicated as $P S L(2, p), \operatorname{PSL}(2,4)$ and $P S L(2,8)$ belong to $V_{3}$ and so, in these cases (E) does not have any solution with the required properties.

Let $q=3^{f}$ or $q=5^{f}$. As $\underset{p}{\mathbb{I}} f$ contains $\underset{p}{\mathbb{F}} d$ for every divisor $d$ of $f$, we may assume that $f$ is a prime. So it remains to consider the cases when $f=2,3$. 
We have

$$
D(x)=\left(x^{2}-1\right)\left(x^{3}+x^{2}+x-1\right)\left(x^{3}-x^{2}+x+1\right),
$$

where the cubic factors are irreducible over $\mathbb{F}_{3}$ and $\mathbb{F}_{5}$. Hence, if $f=3$, there exists $\varepsilon \in \mathbb{F}_{q}, \varepsilon^{2} \neq 0, \pm 1$, such that $D(\varepsilon)=0$.

Now, let $\varepsilon_{1} \in \mathbb{F}_{9}$ be a root of the polynomial $x^{2}+X-1$. Then $D\left(\varepsilon_{1}\right)=-\varepsilon_{1}^{2}$. As -1 is a square in $\mathbb{F}_{9}, D\left(\varepsilon_{1}\right)$ is a square. Moreover, $\varepsilon_{1}^{2}=-\varepsilon_{1}+1$ implies $\varepsilon_{1}^{2} \neq 0, \pm 1$. similarly, if $\varepsilon_{2} \in \mathbb{F}_{25}$ is a root of $x^{2}-X+1$, then $D\left(\varepsilon_{2}\right)=4$ and $\varepsilon_{2}^{2} \neq 0, \pm 1$.

The following result completes the proof of the Theorem.

LEMMA 3. Let $q=2^{f}$, where $f \geq 4$. Then (E) has a solution $u, \in \in \mathbb{I F}_{q}$, such that $\varepsilon \neq 0,1$.

Proof. In characteristic 2 equation (E) reads as follows:

$$
(1+\varepsilon)^{2} u^{2}+(1+\varepsilon)^{4} u+\varepsilon^{2}=0 \text {. }
$$

Let $\varepsilon \neq 1$. Setting $u=y(1+E)^{2}$ the solubility of (E) is equivalent to the solubility of

$$
y^{2}+y+\mu(\varepsilon)=0 \text {, where } \mu(\varepsilon)=\varepsilon^{2} /(1+\varepsilon)^{6} .
$$

Now, by Hilbert's Theorem $90[4$, p. 215] this is equivalent to showing that there exists $\varepsilon$ with $\operatorname{Tr}(\mu(\varepsilon) \cdot)=0$. Let $\varepsilon=\varepsilon_{1}^{-1}+1$. Then $\mu(\varepsilon)=\varepsilon_{1}^{4}+\varepsilon_{1}^{6}$ and so

$$
\operatorname{Tr}(\mu(\varepsilon))=\operatorname{Tr}\left(\varepsilon_{1}^{4}\right)+\operatorname{Tr}\left(\varepsilon_{1}^{6}\right)=\operatorname{Tr}\left(\varepsilon_{1}\right)+\operatorname{Tr}\left(\varepsilon_{1}^{3}\right),
$$

since $\operatorname{Tr}(\alpha)=\operatorname{Tr}\left(\alpha^{2}\right), \alpha \in \mathbb{F}_{q}$. Hence if (E) cannot be solved, then

$$
\operatorname{Tr}(\alpha)+\operatorname{Tr}\left(\alpha^{3}\right)=1, \text { for all } \alpha \neq 0,1
$$

and so

$$
g(x)=\left(x^{2}+x\right)\left(\operatorname{Tr}(x)+\operatorname{Tr}\left(x^{3}\right)+1\right)
$$


would be zero on $I F$. Hence, $g(x)$ would be divisible by $x^{q}+x$. We now show that this is not the case if $f \geq 4$. We have

$$
g(x)=\left(x^{2}+x\right)\left(1+x+x^{3}+\ldots+x^{2^{i}}+x^{3.2^{i}}+\ldots+x^{2^{f-1}}+x^{3.2^{f-1}}\right) .
$$

As the degree of $g(x)$ is less than $2 q-1=2^{f+1}-1$, for $f \geq 3$, it is sufficient to consider the coefficients of $x^{q}$ and $x$. If we can show that these are different, then it is clear that $g(x)$ is not divisible by $x^{q}+x$. Now, every exponent occuring in $g(x)$ equals 1 or is of the form

$$
2^{i}+1,2^{i}+2,3.2^{i}+1,3.2^{i}+2 \text {, for some } 0 \leq i \leq f-1 \text {. }
$$

If $i \geq 2$, then all of these numbers are congruent to 1 or 2 (mod 4), and if $i=0,1$, then these numbers are equal to $2,3,4,5$, 7, 8. As $q \geq 16$, the coefficient of $x^{q}$ is zero. As the coefficient of $x$ equals 1 , the conclusion follows.

\section{References}

[1] R. Brandl, "On groups with small Engel depth", BuZl. Austral. Math. Soc. 28 (1983), 101-110.

[2] N. D. Gupta, "Some group laws equivalent to the commutative law", Arch. Math. (Base Z) 17 (1966), 97-102.

[3] H. Heineken and P.M. Neumann, "Identical relations and decision procedures for groups", J. Austral. Math. Soc. 7 (1967), 39-47.

[4] S. Lang, Algebra, (Addison-Wesley, Reading, Massachusetts 1971).

[5] D. B. Nikolova, "Groups with a two-variable commutator identity", R. Bulgare Sci. 36 (1983), 721-724.

[6] W. M. Schmidt, "A lower bound for the number of solutions of equations over finite fields", $J$. Number Theory 6 (1974) 448-480.

Mathematisches Institut

der Universität,

Am Hubland 12,

D-8700 wurzburg

BRD
Mathematical Institute Bulgarian Academy of Sciences str."Acad.G.Bonchev", bl.8, 1113 Sofia

Bulgaria. 